\title{
Prolonged resuscitation of metabolic acidosis after trauma is associated with more complications
}

\author{
Douglas S. Weinberg ${ }^{1 *}$, Arvind S. Narayanan², Timothy A. Moore ${ }^{1}$ and Heather A. Vallier ${ }^{1}$
}

\begin{abstract}
Background: Optimal patterns for fluid management are controversial in the resuscitation of major trauma. Similarly, appropriate surgical timing is often unclear in orthopedic polytrauma. Early appropriate care (EAC) has recently been introduced as an objective model to determine readiness for surgery based on the resuscitation of metabolic acidosis. EAC is an objective treatment algorithm that recommends fracture fixation within $36 \mathrm{~h}$ when either lactate $<4.0 \mathrm{mmol} / \mathrm{L}$, $\mathrm{pH} \geq 7.25$, or base excess (BE) $\geq-5.5 \mathrm{mmol} / \mathrm{L}$. The aim of this study is to better characterize the relationship between post-operative complications and the time required for resuscitation of metabolic acidosis using EAC.

Methods: At an adult level 1 trauma center, 332 patients with major trauma (Injury Severity Score (ISS) $\geq 16$ ) were prospectively treated with EAC. The time from injury to EAC resuscitation was determined in all patients. Age, race, gender, ISS, American Society of Anesthesiologists score (ASA), body mass index (BMI), outside hospital transfer status, number of fractures, and the specific fractures were also reviewed. Complications in the 6-month post-operative period were adjudicated by an independent multidisciplinary committee of trauma physicians and included infection, sepsis, pulmonary embolism, deep venous thrombosis, renal failure, multiorgan failure, pneumonia, and acute respiratory distress syndrome. Univariate analysis and binomial logistic regression analysis were used to compare complications between groups.
\end{abstract}

Results: Sixty-six patients developed complications, which was less than a historical cohort of 1,441 patients (19.9\% vs. $22.1 \%)$. ISS $(p<0.0005)$ and time to EAC resuscitation $(p=0.041)$ were independent predictors of complication rate. A 2.7-h increase in time to resuscitation had odds for sustaining a complication equivalent to a 1-unit increase on the ISS.

Conclusions: EAC guidelines were safe, effective, and practically implemented in a level 1 trauma center. During the resuscitation course, increased exposure to acidosis was associated with a higher complication rate. Identifying the innate differences in the response, regulation, and resolution of acidosis in these critically injured patients is an important area for trauma research.

Level of evidence: Level 1: prognostic study.

Keywords: Early appropriate care, Acidosis, Fixation timing, Resuscitation, Polytrauma

\footnotetext{
* Correspondence: Dsw56@case.edu

'Department of Orthopaedic Surgery, MetroHealth Medical Center, Case Western Reserve University, 2500 MetroHealth Dr., Cleveland, OH 44109, USA Full list of author information is available at the end of the article
} 


\section{Background}

The timing of osteosynthesis in multiple-injured patients is controversial. Many authors have promoted damage control orthopedics (DCO), an initially conservative approach for severely injured patients as a means to decrease postoperative complications [1-6]. However, this comes at the expense of providing early definitive fracture care, which improves posture, accelerates ambulation, and reduces complications related to inactivity [7-15]. While early definitive fixation and DCO both offer undeniable benefits in certain clinical situations, establishing appropriate patient criterion remains elusive.

Recently, early appropriate care (EAC) was introduced as an objective algorithm to determine surgical readiness. EAC quantifies the resuscitation of metabolic acidosis to enhance patient selection between early total care and DCO. Statistical modeling showed that complications are decreased when definitive fixation occurred within $36 \mathrm{~h}$ of injury with at least one of the following: lactate $<4.0 \mathrm{mmol} / \mathrm{L}, \mathrm{pH} \geq 7.25$, or base excess $(\mathrm{BE}) \geq-5.5 \mathrm{mmol} / \mathrm{L}$. This protocol offers the additional advantages of being simple, easily applicable to every trauma patient, and has been designed and reviewed by a multidisciplinary team of trauma providers [16].

However, the simplicity afforded by the EAC guidelines does not incorporate timing over which the resuscitation of acidosis occurs. With the abundance of recent literature emphasizing the importance of optimal fluid management, it would therefore be clinically prudent to determine if the timing to resuscitation in patients managed with EAC affected post-operative complication rate [17-20]. Consequently, we designed a prospective study to investigate the relationship between the EAC resuscitation course and the development of post-operative complications.

\section{Methods \\ Implementing early appropriate care at a level 1 trauma center}

Following institutional review board (MetroHealth Institutional Review Board) approval, patients were prospectively managed with early appropriate care from October 2010 through March 2013 at a level 1 adult trauma center. EAC treatment guidelines recommended definitive fixation of the orthopedic fractures within $36 \mathrm{~h}$ of injury once resuscitation of initial acidosis had improved such that lactate $<4.0 \mathrm{mmol} / \mathrm{L}, \mathrm{pH} \geq 7.25$, or $\mathrm{BE} \geq-5.5 \mathrm{mmol} / \mathrm{L}$ [16]. Definitive fixation was defined as an orthopedic intervention was intended to represent final treatment.

Patients were excluded for having injuries sustained from low-energy mechanisms (252), being skeletally immature (23), or having an Injury Severity Score (ISS) $<16$ (190). Patients with definitive nonoperative treatment were also excluded (835). Patients who received definitive orthopedic fixation before being appropriately resuscitated were excluded (three). One thousand one hundred and seventy-eight patients were excluded in total, and three hundred and thirty-two adult patients were enrolled.

\section{Timing to resuscitation}

The time of injury was available in all 332 patients enrolled. A lactate value was ordered on all patients in the initial trauma workup, and arterial blood gas analysis was obtained at the discretion of the attending general trauma surgeon. Lab values were repeated in 8-h increments until they had normalized. All patients were off presser support at the time of surgery. The time from injury to EAC protocol resuscitation was recorded. Other data obtained for all patients included age, race, gender, ISS, American Society of Anesthesiologists score (ASA), body mass index (BMI), outside hospital transfer status, types of fractures, and types and severity of injury to other systems.

\section{Measurements of outcome}

Complications in the 6-month post-operative period were determined by an independent adjudication committee that was not involved in the development of the EAC protocol or any of its earlier research. The committee consisted of physicians from a multidisciplinary background, including those specializing in general trauma surgery/critical care, orthopedic surgery, neurosurgery, and anesthesiology. Patients were assessed for acute renal failure [21], multiple organ failure [22], sepsis [23], deep venous thrombosis (DVT) [21], infection, and pulmonary complications: pneumonia [24], acute respiratory distress syndrome (ARDS) [25], and pulmonary embolism (PE) [26].

\section{Statistical analysis}

Multiple regression analysis was performed to determine the independent predictors of time required to EAC protocol resuscitation. All univariate data was analyzed for skewness and kurtosis. Overall complication rate was compared to a historical cohort. Comparisons between groups were performed with the independent samples Mann-Whitney $U$ test, or independent samples $t$ test for continuous variables, where parametrically appropriate. The Fisher's exact test was used for categorical variables. Stepwise binomial logistic regression with backward selection was performed to identify independent clinical predictors of complication rate, with the same group of comparisons that were performed for the univariate analysis. Variables with a $p$ value of less than 0.20 in univariate comparison were chosen as candidates for the binomial logistic model, with significance determined with use of the likelihood ratio chi-square test. Significance was set at a $p$ value of less than 0.05 in regression testing. Adjusted odds ratios and $95 \%$ confidence intervals were derived. Receiver operating curves were generated. Statistical analysis was performed with the 
SPSS (version 22.0; IBM Incorporated, Armonk, NY) software package. Significance was set at $p \leq 0.05$.

\section{Results}

\section{Descriptive data}

Three hundred and thirty-two patients were prospectively assessed and treated according to EAC criteria, including 96 females $(28.9 \%)$ and 236 males with an average age of $39 \pm 16$ years. Mean BMI was $29.6 \pm 7.8$, and mean ISS was $27 \pm 12$. There were a total of 376 fractures in these patients (1.14 fractures/patient) with 171 fractures of the femur, 56 fractures of the acetabulum, 70 fractures of the pelvic ring, six fractures of the cervical spine, and 73 fractures of the thoracolumbar spine. The mean time required to achieve EAC resuscitation goal was $6.8 \pm 7.8 \mathrm{~h}$ (range, 0.3 to 35.3 ). Sixty-six patients sustained at least one post-operative complication (19.9\%); while 266 did not have any complications, this was slightly less than a historical control of 1443 patients with a $22.1 \%$ complication rate $(p=0.155)$.

Transfer from an outside hospital was an independent predictor of more time needed to achieve EAC resuscitation, requiring an additional $1 \mathrm{~h}$ and $10 \mathrm{~min}$ of resuscitation time on average (standardized beta 0.112, $p=0.045$ ). The time required for EAC resuscitation was independent of age, race, gender, ISS, ASA, BMI, number of fractures, and any specific fractures (Table 1). All correlations in the multiple regression analysis were below 0.5. All VIF values were below 10 and coefficient tolerances above 0.10 . Normal probability plots of the regression standardized residual

Table 1 Multiple regression results, independent predictors of time to EAC resuscitation

\begin{tabular}{|c|c|c|}
\hline Independent variable & $\begin{array}{l}\text { Standardized } \\
\text { beta }^{a}\end{array}$ & Significance \\
\hline Age & 0.030 & 0.596 \\
\hline African-American race & 0.070 & 0.511 \\
\hline Gender & -0.005 & 0.935 \\
\hline Injury Severity Score (ISS) & -0.005 & 0.935 \\
\hline $\begin{array}{l}\text { American Society of Anesthesiologists } \\
\text { score (ASA) }\end{array}$ & -0.039 & 0.578 \\
\hline Body mass index (BMI) & 0.018 & 0.753 \\
\hline Transfer from outside hospital (OSH) & 0.112 & $0.045+$ \\
\hline Number of fractures & -0.111 & 0.296 \\
\hline Presence of a femur fracture & -0.036 & 0.735 \\
\hline Presence of an acetabular fracture & 0.029 & 0.736 \\
\hline Presence of a pelvic ring fracture & 0.075 & 0.403 \\
\hline Presence of a cervical spine fracture & 0.096 & 0.089 \\
\hline $\begin{array}{l}\text { Presence of a thoracolumbar spine } \\
\text { fracture }\end{array}$ & 0.065 & 0.712 \\
\hline $\begin{array}{l}t p<0.05 \\
\text { aA positive value for race suggests that } \\
\text { receive resuscitation. A positive value for } \\
\text { longer to receive resuscitation. A positiv } \\
\text { hospital indicates that outside transfer to }\end{array}$ & 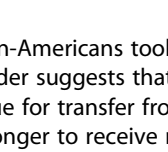 & $\begin{array}{l}\text { er to } \\
\text { les took } \\
\text { side } \\
\text { tation }\end{array}$ \\
\hline
\end{tabular}

were inspected for normality. Scatterplots of the residuals were inspected for homoscedasticity, and a Cook's distance $<1$ confirmed any undue influence from outliers.

\section{Univariate analysis}

The 66 patients who developed a post-operative complication had higher ISS scores $(p<0.0005)$, higher ASA scores $(p<0.0005)$, significantly increased time to EAC resuscitation $(p=0.022$, and were more likely to have pelvic ring $(p=0.027)$, and thoracolumbar spine $(p=0.045)$ fractures. Patients with femur fractures had fewer complications than those with other fractures $(p=0.005)$, and female patients showed a trend towards decreased complications $(p=0.070)$ (Table 2).

\section{Multivariate analysis}

Four independent predictors of complications were identified (Table 3). Complications were fewer in females (odds ratio $[\mathrm{OR}]=0.428,95 \%$ confidence interval $[\mathrm{CI}]$ 0.202$0.908, p=0.027$ ). Every 1 -point increase on the ISS scale was associated with a $8.7 \%$ increase in the odds of sustaining a complication $([\mathrm{OR}]=1.087,[\mathrm{CI}] 1.061-1.118, p<$ 0.0005). For each $1-\mathrm{h}$ increase in the time required to reach EAC resuscitation, there was a $3.2 \%$ increase in the odds of sustaining a complication $([\mathrm{OR}]=1.032$, [CI] 1.001-1.063, $p=0.041)$. The presence of a femur fracture was a negative predictor of complications $([\mathrm{OR}]=0.447,[\mathrm{CI}] 0.236-0.845, p=0.013)$.

ASA score, the presence of a pelvic ring injury, and the presence of a thoracolumbar spine fracture were not independent predictors of sustaining a complication. The logistic regression model was statistically significant, $X^{2}(4)=64.718, p<0.0005$. All correlations were below 0.5 . All VIF values were below 10 and coefficient tolerances above 0.10 . Scatterplots of the residuals were inspected for homoscedasticity, and a Cook's distance $<1$ confirmed any undue influence from outliers.

\section{Cutoff points for time resuscitation}

In the continued design and practical application of EAC, it may be advantageous to categorize timing of resuscitation into cutoff hours. The different cutoff points extrapolated from the receiver operating curves suggested that there was no clear time during EAC resuscitation after which point the risk of having a complication increased markedly (Table 4). This confirmed the linear relationship between timing to EAC resuscitation and complication risk. A cutoff value of $12 \mathrm{~h}$ was chosen to plot the values in Fig. 1 .

\section{Discussion}

Resuscitation is of paramount importance in the management of trauma patients. Historically, this proceeded with intravenous fluid with a principal goal of normalizing hemodynamic status [17, 27-29]. However, critical care 
Table 2 Univariate analysis for patients with post-operative complications

\begin{tabular}{|c|c|c|c|}
\hline \multirow[t]{2}{*}{ Variable } & \multirow{2}{*}{$\begin{array}{l}\text { No complication }^{a} \\
n=266(80.1 \%)\end{array}$} & \multirow{2}{*}{$\begin{array}{l}\text { Complication }^{\mathrm{a}} \\
n=66(19.9 \%)\end{array}$} & \multirow[t]{2}{*}{ Significance } \\
\hline & & & \\
\hline Age (years) & $39.1 \pm 16.2$ & $40.3 \pm 17.3$ & 0.597 \\
\hline African-American race & $53(19.7 \%)$ & 15 (22.7 \%) & 0.505 \\
\hline Female gender & $82(31.1 \%)$ & $13(19.7 \%)$ & 0.070 \\
\hline ISS & $24.5 \pm 9.3$ & $36.4 \pm 15.0$ & $<0.0005+$ \\
\hline ASA score & $2.7 \pm 0.8$ & $3.3 \pm 0.9$ & $0.020+$ \\
\hline BMI & $29.5 \pm 7.9$ & $30.3 \pm 7.7$ & 0.483 \\
\hline OSH transfer & $113(42.5 \%)$ & $30(45.5 \%)$ & 0.679 \\
\hline Time to EAC resuscitation (hours) & $6.29 \pm 8.24$ & $9.07 \pm 10.58$ & $0.022+$ \\
\hline Number of fractures ${ }^{b}$ & $1.1 \pm 0.4$ & $1.2 \pm 0.4$ & 0.331 \\
\hline Presence of a femur fracture ${ }^{c}$ & $138(51.9 \%)$ & $21(31.8 \%)$ & $0.005+$ \\
\hline Presence of an acetabulum fracture & $46(17.3 \%)$ & 10 (15.2\%) & 0.854 \\
\hline Presence of a pelvic ring fracture & 49 (18.4\%) & $21(31.8 \%)$ & $0.027 \dagger$ \\
\hline Presence of a cervical spine fracture & $3(1.1 \%)$ & $3(4.5 \%)$ & 0.096 \\
\hline Presence of a thoracolumbar spine fracture & $52(19.5 \%)$ & $21(31.8 \%)$ & $0.045 \dagger$ \\
\hline
\end{tabular}

ISS Injury Severity Score, ASA American Society of Anesthesiologists' Classification, BMI body mass index, OSH outside hospital transfer $+p<0.05$

${ }^{a}$ The values are given as the mean and the standard deviation for continuous variables and as the number of patients in the respective outcome group, with the percentage in parentheses for categorical variables

${ }^{\mathrm{b}}$ Compared with the chi-square test

${ }^{\mathrm{C}}$ Twelve patients sustained bilateral femur fractures. Since the univariate analysis was designed to assess the presence or absence of a femur fracture, the number of individuals with femur fractures (159) is less than and discordant with the number of femur fractures in total (171)

providers later began to focus on the poor outcomes in patients who became coagulopathic (INR $>1.5$ ), hypoth ermic (temperature $<35{ }^{\circ} \mathrm{C}$ ), and acidotic $(\mathrm{pH}<7.2)$. Rotondo and Zonies described this as the "trauma triad of death" in 1997; this has since prompted trauma systems to investigate a number of different approaches for managing fluid resuscitation [28].

Recent literature has recommended that trauma providers adopt a "less is more" approach in attempts to decrease complications [30]. The American College of Surgeons' Advanced Trauma Life Support (ATLS) guidelines recommend isotonic fluid administration during the resuscitation of adult polytrauma [31]. As Feinman et al. remind us, this only transiently increases intravascular volume: one third of isotonic fluid remains extracellular-of which, only one fourth remains intravascular. The remaining fluid becomes interstitial, with profound consequences on inflammation, microvascular compliance, and cell volume regulatory mechanisms. The resulting tissue edema can provoke acute coronary syndrome, respiratory compromise, and end-organ failure, among other devastating complications [17].

It is therefore not surprising that considerable effort has been devoted to establishing appropriate endpoints for trauma resuscitation. EAC was spawned with this goal in mind: creating an algorithm for the resuscitation of severe orthopedic polytrauma that optimizes surgical timing for fractures of the femur, acetabulum, pelvic ring, and spine

Table 3 Multivariate analysis, binomial logistic regression results, independent predictors of complications

\begin{tabular}{|c|c|c|c|c|c|c|}
\hline \multirow[t]{2}{*}{ Independent variable } & \multirow[t]{2}{*}{ Regression coefficient (B) } & \multirow[t]{2}{*}{ Wald statistic } & \multirow[t]{2}{*}{ Significance } & \multirow[t]{2}{*}{ Odds ratio } & \multicolumn{2}{|c|}{$95 \%$ Cl for odds ratio } \\
\hline & & & & & Lower & Upper \\
\hline Female gender & $-0.848^{\mathrm{a}}$ & 4.896 & $0.027 \dagger$ & 0.428 & 0.202 & 0.908 \\
\hline ISS & 0.084 & 43.957 & $<0.0005 \dagger$ & 1.087 & 1.061 & 1.115 \\
\hline Time to resuscitation & 0.031 & 4.180 & $0.041+$ & 1.032 & 1.001 & 1.063 \\
\hline Presence of femur fracture & $-0.806^{\mathrm{b}}$ & 6.150 & $0.013+$ & 0.447 & 0.236 & 0.845 \\
\hline Constant & -2.696 & 19.090 & $<0.0005$ & 0.067 & & \\
\hline
\end{tabular}

A 2.7-h increase in time to resuscitation had equivalent odds for sustaining a complication as a 1-unit increase on the ISS ISS Injury Severity Score

$+p<0.05$

${ }^{a} \mathrm{~A}$ negative value for gender suggests females suffer fewer complications than males

${ }^{\mathrm{b}} \mathrm{A}$ negative value for femur fracture suggests that patients with a femur fracture suffer fewer complications than those without 
Table 4 Sensitivity, specificity, PPV, NPV, and accuracy for predicting a complication given specific cutoff times during resuscitation course

\begin{tabular}{llllll}
\hline Cutoff time (hours) & Sensitivity (\%) & Specificity (\%) & PPV (\%) & NPV (\%) & Accuracy (\%) \\
\hline 2 & 74 & 26 & 50 & 65 & 50 \\
3 & 70 & 36 & 52 & 54 & 53 \\
6 & 46 & 67 & 57 & 56 & 56 \\
9 & 35 & 82 & 66 & 56 & 58 \\
11.25 & 32 & 86 & 70 & 66 & 51 \\
12 & 26 & 87 & 67 & 51 & 59 \\
18 & 12 & 91 & 96 & 69 & 51 \\
\hline
\end{tabular}

PPV positive predictive value, NPV negative predictive value

[16]. Earlier reports have shown decreased complications compared to historical cohorts. The protocol recommends definitive stabilization within $36 \mathrm{~h}$ if at least one of the following three conditions is met: lactate $<4.0 \mathrm{mmol} / \mathrm{L}$, $\mathrm{pH} \geq 7.25$, or $\mathrm{BE} \geq-5.5 \mathrm{mmol} / \mathrm{L}$. EAC is simple and convenient, and preliminary results suggest that it is safe and effective [32]. However, to our knowledge, there exists limited data regarding the impact of resuscitative delay on post-operative complications using the EAC-or any alternative-resuscitative biomarkers.

In this prospective analysis, the only independent predictor of delay in resuscitation was transfer from an outside hospital. This result is not surprising, as transfer of care in any trauma system inherently introduces delays. Furthermore, there has been a recent trend towards delaying fluid administration for these patients, for many of the reasons discussed above [33]. One may have expected the more critically injured patients to have longer resuscitation courses; however, this was not the case in this cohort or others conducted previously [34].
Predictably, these more critically injured patients had more complications. Tornetta et al. and other authors have suggested that complications in multiple-injured patients with fracture are most strongly associated with the nature of the pre-hospital condition [35]. Our results showed that delays in resuscitation were directly related to complication rate. The most obvious explanation would assume that these patients were more severely injured and required a more complete resuscitation course that resulted in surgical delay-all factors that have been linked to poor outcomes. However, this was not the case: ISS was not a significant predictor of resuscitation time, and moreover, the endpoint of resuscitation was an independent predictor of complication rate.

It is more likely that these complications were direct consequences of prolonged acidosis. In severe polytrauma, acid-base homeostasis is disrupted by inflammatory cytokines. Even with judicious allocation of bicarbonate reserve, the amount available may be insufficient for the compromised homeostatic mechanisms to mount an effective

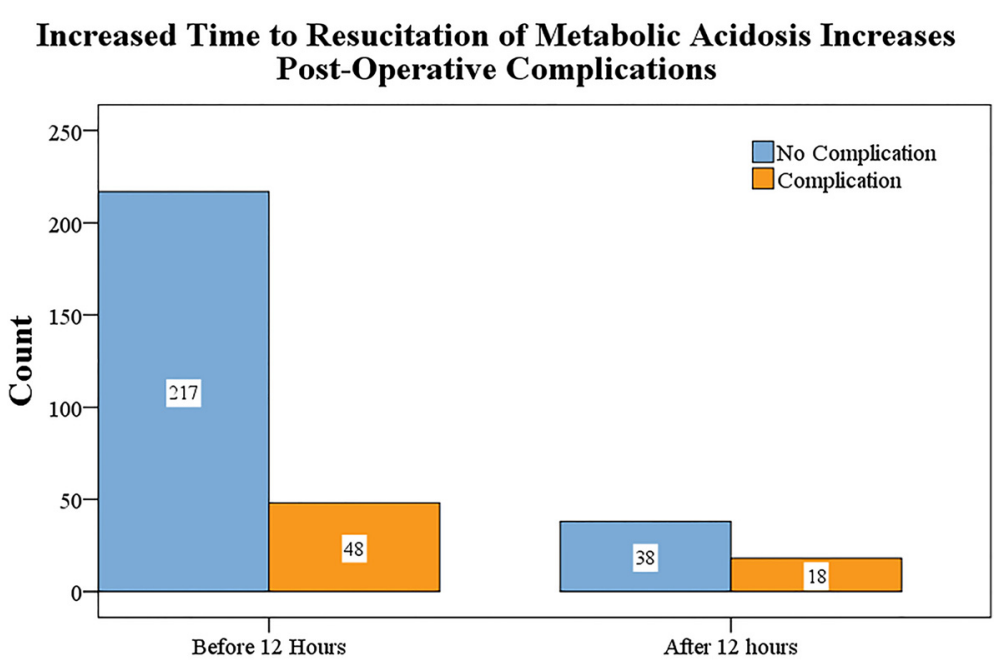

Time to EAC Resucitation

Fig. 1 Number of complications based on time to EAC resuscitation 
response [36]. These severely injured patients with impaired acid-base regulation and prolonged exposure to acidotic environment were then not surprisingly at an increased risk for complications and, in other studies, mortality [37]. Regression modeling confirmed a direct linear relationship between time to EAC protocol resuscitation and complication rate, and while a time point of slightly under $12 \mathrm{~h}$ of resuscitation maximized accuracy of this model, there existed no clear inflection point to suggest establishing a firm cutoff goal for completion of resuscitation. Females were also shown to have decreased complications, perhaps for reasons related to improved response to acid-base perturbations. Previous authors have demonstrated femoral shaft fractures to have lower complication rates than other fractures, perhaps given the comparatively short surgical duration and relatively little associated surgical blood loss [38].

Morshed et al. reviewed a large database of multipleinjured patients with femur fractures and showed that complications were minimized when fixation was performed between $12-24 \mathrm{~h}$ from the time of injury. Since surgical criteria vary widely between trauma networks, the authors inferred that early fracture fixation was a direct surrogate for under-resuscitation. They therefore concluded that incomplete resuscitation was a likely cause of complications [39]. Similarly, while our results show a significant correlation between the time to resuscitation and complications in polytrauma, this study does not establish causation. Cause and effect remain unclear. Moreover, the effects of delayed resuscitation were modest at best compared to injury severity, with a 2.7 -h delay in resuscitation having equivalent odds for sustaining a complication as 1-unit increase on the ISS. This serves as another humbling reminder that although interventions performed in the arena of trauma care are crucial, frequently, patient outcomes are very strongly determined by the nature of their pre-hospital condition. However, as Morshed et al. remind us, fracture fixation represents an essential part of the resuscitation process, especially for certain fractures of the pelvic ring and femur that may be associated with massive hemorrhage.

Going forward, it would be imprudent to advise expedited resuscitation based on this data alone. This experiment made no attempt at quantifying how (or at what rate) EAC resuscitation was to be achieved. In doing so, we were only able to monitor the effective "outputs" of this resuscitation, with little information regarding the "inputs" of treatment. These decisions were made by the general trauma surgery team, who incorporated many of the complicated considerations discussed earlier.

Nonetheless, there clearly exist important differences among individuals in the response to insults of the acid-base system. Identifying these organic differences, and perhaps manipulating them to optimize resuscitation, remains elusive and an important area for future research. This should be especially relevant for patients with existing medical conditions such as diabetes, renal failure, and chronic obstructive pulmonary disease, all of which have impaired regulation of acid-base balance [40]. Others with ischemic heart disease or congestive heart failure may also have physiological limitations making a slightly longer resuscitative course safer and more desirable. This study, and the EAC criterion as a whole, do not account for these or any other medical comorbidities. It would therefore be important to determine which of these conditions were independent predictors of complications and then thoroughly analyze their resuscitation course. This represents an essential area for continued study.

Other important limitations of EAC are that it is exclusively dependent on metabolic acidosis to determine surgical timing. However, the design of EAC affords consideration of multiple lab values, allowing one to choose biomarkers based on institution-specific order panels, practitioner familiarity, or in consolidation with tests ordered for other purposes. While each of the three resuscitative parameters used are expected to be collinear with one another, this allows versatility in implementation. However, its statistical design may incompletely characterize the relationship between specific injury patterns and biomarkers. While the nature of any universal protocol is appealing to trauma providers, it may be necessary to adjust cutoff parameters based on anticipated blood loss and surgical duration. This study was designed to investigate definitive treatment patterns and did not incorporate the timing of skeletal traction or any other external fixation device; this has been extensively reported previously. Other limitations of EAC have been discussed previously [32]. Similarly, providers should consider other aspects of patient, surgeon, and hospital readiness before surgery. Strengths of the study include a large patient sample size with prospectively collected data, consistencies in patient care, with standardized methods for DVT prophylaxis, perioperative antibiotics, ventilator management, and nutrition. In addition, all patients met the resuscitation criteria within $36 \mathrm{~h}$.

\section{Conclusion}

In summary, resuscitation with EAC criteria has been shown to decrease complications compared to historical cohorts. Definitive osteosynthesis should proceed when one of the following parameters is adequately resuscitated: lactate $<4.0 \mathrm{mmol} / \mathrm{L}, \mathrm{pH} \geq 7.25$, or $\mathrm{BE} \geq-5.5 \mathrm{mmol} / \mathrm{L}$. However, we would recommend that readers rely on their clinical experience to make these decisions based on their physical examination, other laboratory findings, and any operational issues related to their specific trauma system. During the course of resuscitation, prolonged exposure to 
acidosis is associated with a greater risk for complications. Further characterizing the relationships between acidosis, resuscitation course, and complications are required. Early reports such as this spark the need for future study in more patients across multiple institutions.

\section{Consent}

Written informed consent was obtained from the patient for the publication of this report and any accompanying images.

\section{Abbreviations \\ DCO: damage control orthopedics; EAC: early appropriate care.}

\section{Competing interests}

The authors declare that they have no competing interests.

\section{Authors' contributions}

DW designed the study, acquired data, conducted statistical analysis, drafted the manuscript, and critically reviewed the final version of the manuscript. AN acquired data, helped edit the manuscript, and critically reviewed the final version of the manuscript. TM conceptualized the study, organized data, edited the manuscript, and critically reviewed the final version of the manuscript. HV conceptualized the study, organized the research team, designed the study, acquired data, conducted statistical analysis, drafted the manuscript, and critically reviewed the final version of the manuscript. All authors read and approved the final manuscript.

\section{Acknowledgements}

We wish to acknowledge the independent adjudication committee for their help in performing data analysis. This study did not use internal or external funding.

\section{Author details}

'Department of Orthopaedic Surgery, MetroHealth Medical Center, Case Western Reserve University, 2500 MetroHealth Dr., Cleveland, OH 44109, USA. ${ }^{2}$ Case Western Reserve University, School of Medicine, 10900 Euclid Avenue, Cleveland, OH 44106, USA.

\section{Received: 19 August 2015 Accepted: 5 September 2015}

Published online: 24 September 2015

\section{References}

1. Scalea TM, Boswell SA, Scott JD, Mitchell KA, Kramer ME, Pollak AN. External fixation as a bridge to intramedullary nailing for patients with multiple injuries and with femur fractures: damage control orthopedics. J Trauma. 2000:48:613-21.

2. Claridge JA, Crabtree TD, Pelletier SJ, Butler K, Sawyer RG, Young JS. Persistent occult hypoperfusion is associated with a significant increase in infection rate and mortality in major trauma patients. J Trauma. 2000;48:8-14.

3. Harwood PJ, Giannoudis PV, van Griensven M, Krettek C, Pape H-C. Alterations in the systemic inflammatory response after early total care and damage control procedures for femoral shaft fracture in severely injured patients. I Trauma. 2005;58:446-52.

4. Pape H-C, Griensven MV, Hildebrand FF, Tzioupis $\mathrm{CT}$, Sommer KL, Krettek CC, et al. Systemic inflammatory response after extremity or truncal fracture operations. J Trauma. 2008;65:1379-84.

5. Taeger G, Ruchholtz S, Waydhas C, Lewan U, Schmidt B, Nast-Kolb D. Damage control orthopedics in patients with multiple injuries is effective, time saving, and safe. J Trauma. 2005;59:409-16.

6. Nahm NJ, Patterson BM, Vallier HA. The impact of injury severity and transfer status on reimbursement for care of femur fractures. J Trauma Acute Care Surg. 2012;73:957-65.

7. Bone LB, Johnson KD, Weigelt J, Scheinberg R. Early versus delayed stabilization of femoral fractures. A prospective randomized study. J Bone Joint Surg Am. 1989;71:336-40.
8. Johnson KD, Cadambi A, Seibert GB. Incidence of adult respiratory distress syndrome in patients with multiple musculoskeletal injuries: effect of early operative stabilization of fractures. J Trauma. 1985;25:375-84.

9. Lefaivre KA, Starr AJ, Stahel PF, Elliott AC, Smith WR. Prediction of pulmonary morbidity and mortality in patients with femur fracture. J Trauma. 2010;69:1527-35.

10. Nahm NJ, Vallier HA. Timing of definitive treatment of femoral shaft fractures in patients with multiple injuries: a systematic review of randomized and nonrandomized trials. J Trauma Acute Care Surg. 2012;73:1046-63.

11. Vallier HA, Cureton BA, Ekstein C, Oldenburg FP, Wilber JH. Early definitive stabilization of unstable pelvis and acetabulum fractures reduces morbidity. J Trauma. 2010;69:677-84.

12. Schinkel C, Frangen TM, Kmetic A, Andress H-J, Muhr G. Timing of thoracic spine stabilization in trauma patients: impact on clinical course and outcome. J Trauma. 2006;61:156-60.

13. Stahel PF, Flierl MA, Moore EE, Smith WR, Beauchamp KM, Dwyer A. Advocating "spine damage control" as a safe and effective treatment modality for unstable thoracolumbar fractures in polytrauma patients: a hypothesis. J Trauma Manag Outcomes. 2009;3:6.

14. Brundage SI, McGhan R, Jurkovich GJ, Mack CD, Maier RV. Timing of femur fracture fixation: effect on outcome in patients with thoracic and head injuries. J Trauma. 2002;52:299-307.

15. Durham RM, Moran JJ, Mazuski JE, Shapiro MJ, Baue AE, Flint LM. Multiple organ failure in trauma patients. J Trauma. 2003;55:608-16.

16. Vallier HA, Wang X, Moore TA, Wilber JH, Como JJ. Timing of orthopaedic surgery in multiple trauma patients: development of a protocol for early appropriate care. J Orthop Trauma. 2013;27:543-51.

17. Feinman $M$, Cotton $B A$, Haut ER. Optimal fluid resuscitation in trauma: type, timing, and total. Curr Opin Crit Care. 2014;20:366-72.

18. Kwan I, Bunn F, Chinnock P, Roberts I. Timing and volume of fluid administration for patients with bleeding. The Cochrane Library. 2014.

19. Duchesne JC, Pereira BM, Fraga GP. Damage control resuscitation. Trauma Surgery: Springer; 2014. p. 27-42.

20. Maier RV. A century of evolution in trauma resuscitation. J Am Coll Surg. 2014;219:335-45.

21. Bellomo R, Ronco C, Kellum JA, Mehta RL, Palevsky P. Acute renal failure-definition, outcome measures, animal models, fluid therapy and information technology needs: the Second International Consensus Conference of the Acute Dialysis Quality Initiative (ADQI) Group. Crit Care. 2004;8:204-12

22. Bone RC, Balk RA, Cerra FB, Dellinger RP, Fein AM, Knaus WA, et al. Definitions for sepsis and organ failure and guidelines for the use of innovative therapies in sepsis. The ACCP/SCCM Consensus Conference Committee. American College of Chest Physicians/Society of Critical Care Medicine. Chest J. 1992;101:1644-55.

23. Dellinger RP, Levy MM, Rhodes A, Annane D, Gerlach H, Opal SM, et al. Surviving Sepsis Campaign: international guidelines for management of severe sepsis and septic shock: 2008. Crit. Care Med. 2008;36:296-327.

24. American Thoracic Society, Infectious Diseases Society of America. Guidelines for the management of adults with hospital-acquired, ventilatorassociated, and healthcare-associated pneumonia. Am J Respir Crit Care Med. 2005;171(4):388-416.

25. Bernard GR, Artigas A, Brigham KL, Carlet J, Falke K, Hudson L, et al. The American-European Consensus Conference on ARDS. Definitions, mechanisms, relevant outcomes, and clinical trial coordination. Am J Respir Crit Care Med. 1994;149:818-24.

26. Remy-Jardin M, Remy J, Deschildre F, Artaud D, Beregi JP, Hossein-Foucher $C$, et al. Diagnosis of pulmonary embolism with spiral $\mathrm{CT}$ : comparison with pulmonary angiography and scintigraphy. Radiology. 1996;200:699-706.

27. Ho AM-H, Karmakar MK, Dion PW. Are we giving enough coagulation factors during major trauma resuscitation? Am J Surg. 2005;190:479-84.

28. Rotondo MF, Zonies DH. The damage control sequence and underlying logic. Surg Clin North Am. 1997;77:761-77.

29. Kutcher ME, Howard BM, Sperry JL, Hubbard AE, Decker AL, Cuschieri J, et al. Evolving beyond the vicious triad: differential mediation of traumatic coagulopathy by injury, shock, and resuscitation. J Trauma Acute Care Surg. 2015;78:516-23.

30. Dries D. Fluid resuscitation: less is more*. Crit Care Med. 2014;42:1005-6.

31. Trauma ACOSCO. ATLS, advanced trauma life support for doctors: student course manual: American college of surgeons; 2012. 
32. Vallier HA MT, Como JJ, Wilczewski PA, Steinmetz MP, Wagner KG, Smith CE, et al. Pulmonary complications are reduced with a protocol to standardize timing of fixation based on response to resuscitation. Selected as a "Highlight Paper" from this meeting. Orthopaedic Trauma Association Annual Meeting Minneapolis, MNOctober 2012.

33. Coppola S, Froio S, Chiumello D. Fluid resuscitation in trauma patients: what should we know? Curr Opin Crit Care. 2014;20:444-50.

34. LOWE DK, HEDGES JR, MARBY DW, MENDELSON D. An assessment of time following trauma resuscitation: the transitional evaluation and monitoring phase. J Trauma Acute Care Surg. 1991;31:1265-70.

35. Tornetta P, Mostafavi H, Riina J, Turen C, Reimer B, Levine R, et al. Morbidity and mortality in elderly trauma patients. J Trauma Acute Care Surg. 1999;46:702-6.

36. Giannoudis PV, Smith RM, Bellamy MC, Morrison JF, Dickson RA, Guillou PJ. Stimulation of the inflammatory system by reamed and unreamed nailing of femoral fractures. An analysis of the second hit. J Bone Joint Surg. 1999;81:356-61.

37. Odom SR, Howell MD, Silva GS, Nielsen VM, Gupta A, Shapiro NI, et al. Lactate clearance as a predictor of mortality in trauma patients. J Trauma Acute Care Surg. 2013;74:999-1004.

38. Schutz M, Ruedi T, Tornetta P. Rockwood and Green fractures in adults. 2014.

39. Morshed S, Miclau T, Bembom O, Cohen M, Knudson MM, Colford JM. Delayed internal fixation of femoral shaft fracture reduces mortality among patients with multisystem trauma. J Bone Joint Surg Am. 2009;91:3-13.

40. Adrogué HJ, Madias NE. Management of life-threatening acid-base disorders. New Engl J Med. 1998;338:26-34.

\section{Submit your next manuscript to BioMed Central and take full advantage of:}

- Convenient online submission

- Thorough peer review

- No space constraints or color figure charges

- Immediate publication on acceptance

- Inclusion in PubMed, CAS, Scopus and Google Scholar

- Research which is freely available for redistribution 